\title{
Antenna Selection in Keyhole Channels
}

\author{
Shahab Sanayei, Member, IEEE, and Aria Nosratinia, Senior Member, IEEE
}

\begin{abstract}
This letter presents two results for antenna selection under keyhole condition. First, we analyze the capacity of the antenna-selection keyhole channel. We show that in an $M \times N$ system a small number of selected antennas can match the capacity of a baseline full-antenna system (baseline system has no feedback). Second, we formally prove the intuitive result, until now unproven, that antenna selection in the keyhole multiple-input multiple-output channel preserves the available diversity of the channel.
\end{abstract}

Index Terms-Antenna selection, capacity, diversity order, keyhole channel.

\section{INTRODUCTION}

$\mathbf{T}$ HE PERFORMANCE of a multiple-input multiple-output (MIMO) channel is severely degraded under the so-called keyhole or pinhole effect [1], [2]. Under this condition, the channel loses its spatial degrees of freedom, and the channel matrix becomes rank-deficient [3]. This can happen even when the MIMO component channels are uncorrelated [1]. Under the keyhole condition, the capacity scaling of the MIMO channel, with respect to signal-to-noise ratio (SNR), is no better than a single-input single-output (SISO) channel.

Thus, under the keyhole condition, MIMO coding techniques cannot yield the same impressive capacities that are available in rich scattering environments. With the decreased performance, one naturally wishes to use simpler techniques and hardware, thus motivating our study of antenna selection in the keyhole channel.

MIMO transmitters and receivers require multiple radio frequency (RF) chains and low-noise amplifiers (LNA); antenna selection reduces this costly hardware requirement [4]. Furthermore, multiple antennas generate multiple data paths, requiring high-dimensional signaling and decoding algorithms that impose considerable computational complexity on the system. Antenna selection reduces computational cost as well as hardware cost.

This letter has two components. First, we study the capacity of the antenna-selection keyhole channel, and determine the number of selected antennas (equivalently, the number of RF chains needed) so that we can match the capacity of a baseline system without antenna selection. Second, we prove a result that

Paper approved by V. A. Aalo, the Editor for Diversity and Fading Channel Theory of the IEEE Communications Society. Manuscript received January 17, 2005; revised October 5, 2005.

S. Sanayei is with ArrayComm LLC, San Jose, CA 95131 USA (e-mail: sanayei@ieee.org).

A. Nosratinia is with the Department of Electrical Engineering, University of Texas at Dallas, Richardson, TX 75083 USA (e-mail: aria@utdallas.edu).

Digital Object Identifier 10.1109/TCOMM.2007.892442 was widely believed to be true, but remained unproven: that antenna selection does not decrease the diversity of the keyhole channel. ${ }^{1}$

We use the following notation: $\mathbb{E}[\cdot]$ refers to expected value of a random variable, $\mathbf{h}(i)$ denotes the $i$ th element of the vector $\mathbf{h} \in \mathbb{C}^{N}$. Gamma function is defined as $\Gamma(x)=\int_{0}^{\infty} t^{x-1} e^{-t} d t$. Asymptotic equivalence of $f(\cdot)$ and $g(\cdot)$ is denoted by $f(x) \stackrel{\circ}{=} g(x)$ and defined as follows. If there exist nonzero constants $k_{1}$ and $k_{2}$ such that for small enough $x$ we have $k_{1} \leq|f(x) / g(x)| \leq k_{2}$, we say $f$ and $g$ are equivalent in the asymptote of small values of $x$.

\section{SySTEM MOdEL}

We assume a narrowband frequency-flat linear time-invariant fading channel, with $M$ transmit and $N$ receive antennas. The channel matrix denoted by $\mathbf{H}$ is an $M \times N$ matrix in $\mathbb{C}^{M \times N}$, whose $i j$ th element represents the gain between $i$ th transmit antenna and the $j$ th receive antennas. Under the keyhole condition, the rank of the channel reduces to one. A general model for the keyhole channel is of the following form:

$$
\mathbf{H}=\mathbf{h}_{r} \mathbf{h}_{t}^{T}
$$

where $\mathbf{h}_{t} \in \mathbb{C}^{M}$ and $\mathbf{h}_{r} \in \mathbb{C}^{N}$ are column vectors of the size $M \times 1$ and $N \times 1$, respectively, and each consist of independent and identically distributed (i.i.d.) elements distributed as $\mathcal{C N}(0,1)$. Furthermore, $\mathbf{h}_{r}$ and $\mathbf{h}_{t}$ are assumed to be independent. We assume transmission power is limited and that the received SNR is denoted by $\rho$. We also assume a low-rate but reliable feedback path from the receiver to the transmitter is available.

\section{ANTENNA SELECTION}

In the antenna-selection problem, we assume that the transmitter (also the receiver) selects one or more of the available antennas. Beyond this, the transmitter does not have any additional information, thus the power will be equally distributed among the selected antennas. Assuming equal power splitting among antennas, the capacity of the full keyhole channel (without any antenna selection) is

$$
C=\log \operatorname{det}\left(I_{N}+\frac{\rho}{M} \mathbf{H}^{T} \mathbf{H}\right) .
$$

Since in the keyhole channel, the channel matrix $\mathbf{H}$ has rank 1, we can write, with a small algebraic manipulation

$$
C=\log \left(1+\frac{\rho}{M}\left\|\mathbf{h}_{t}\right\|^{2}\left\|\mathbf{h}_{r}\right\|^{2}\right)
$$

\footnotetext{
${ }^{1}$ We compare antenna selection with a full system that uses all antennas, but has no channel state information (CSI) feedback.
} 
thus, the instantaneous SNR, normalized by the received SNR, can be defined as

$$
\gamma \triangleq \frac{\left\|\mathbf{h}_{t}\right\|^{2}}{M}\left\|\mathbf{h}_{r}\right\|^{2}
$$

and the capacity (conditioned on $\mathbf{H})$ is $C=\log (1+\rho \gamma$ ), which is a monotonic function of SNR.

Since the random variables $\left\|\mathbf{h}_{t}\right\|^{2}$ and $\left\|\mathbf{h}_{r}\right\|^{2}$ are distributed chi-square $\chi_{2 M}^{2}$ and $\chi_{2 N}^{2}$, respectively, the average normalized SNR for the keyhole channel is

$$
\mathbb{E}[\gamma]=N .
$$

Now considering the channel with selection, the selected (sub)channel can be represented as $\widetilde{\mathbf{H}}_{L \times K}=\widetilde{\mathbf{h}}_{r} \widetilde{\mathbf{h}}_{t}^{T}$, where $\widetilde{\mathbf{h}}_{t} \in \mathbb{C}^{k}$ and $\widetilde{\mathbf{h}}_{r} \in \mathbb{C}^{L}$ are the channel vectors of selected antennas at the transmit and receive sides. Power is split equally between selected transmit antennas. The normalized instantaneous SNR for the selected channel is

$$
\widetilde{\gamma}=\frac{\left\|\widetilde{\mathbf{h}}_{t}\right\|^{2}}{K}\left\|\widetilde{\mathbf{h}}_{r}\right\|^{2}
$$

and the capacity formula is $C=\log (1+\rho \widetilde{\gamma})$. In order to maximize the capacity subject to selection, it suffices to maximize $\widetilde{\gamma}$. An important consequence of (6) is that joint transmit and receive antenna selection in the keyhole channel decouples into MISO and SIMO antenna selection problems.

The average per-element energy of a vector is less than the energy of the largest vector element, that is

$$
\frac{\left\|\widetilde{\mathbf{h}}_{t}\right\|^{2}}{K} \leq \max _{i}\left|\mathbf{h}_{t}(i)\right|^{2} .
$$

Therefore the best strategy is to select only one antenna at the transmitter, the one with the highest channel gain. ${ }^{2}$ Transmitantenna selection is possible with minimal feedback of $\lceil\log M\rceil$ bits. For $K=1$ and $L=N$, it can be easily shown that

$$
\mathbb{E}[\widetilde{\gamma}]=N \sum_{j=1}^{M} \frac{1}{j} .
$$

The above harmonic sum, when compared with (5), characterizes the gain of transmit-antenna selection over the full channel without CSI.

We now proceed to the receive-side selection. The diversity obtained by selecting $L$ out of $N$ antennas is known as generalized selection diversity [5], and has been extensively studied in the literature. Using results from [5] we can calculate the average normalized SNR for antenna selection with $K=1$ and arbitrary $L$

$$
\begin{aligned}
\mathbb{E}[\widetilde{\gamma}] & =\sum_{k=1}^{M} \frac{1}{k}\left(L+L \sum_{j=L+1}^{N} \frac{1}{j}\right) \\
& =g(L) .
\end{aligned}
$$

${ }^{2}$ With complete CSI at the transmitter, it is possible to do even better via beamforming. However, that requires significant feedback rate as well as a more complex transmitter.
TABLE I

$M \times N$ MIMO SySTEM With ANTENNA SELECTION. TABLE SHOWS THE Number of ReCEIVE ANTENNAS SELECTED (TOGETHER With ONE SELECTED TX ANTENNA) TO Match CAPACITY OF BASELINE $M \times N$ SYSTEM WITH NO TX CSI

\begin{tabular}{c|ccccccccc|}
\cline { 2 - 9 } Baseline Tx Antennas & \multicolumn{10}{c|}{ Baseline Rx Antennas } \\
\hline 2 & 2 & 3 & 4 & 5 & 6 & 7 & 8 & 9 & 10 \\
\hline 3 & 1 & 1 & 1 & 2 & 2 & 2 & 2 & 3 & 3 \\
4 & 1 & 1 & 1 & 1 & 2 & 2 & 2 & 2 & 2 \\
5 & 1 & 1 & 1 & 1 & 1 & 1 & 1 & 2 & 2 \\
6 & 1 & 1 & 1 & 1 & 1 & 1 & 1 & 1 & 2 \\
7 & 1 & 1 & 1 & 1 & 1 & 1 & 1 & 1 & 1 \\
\hline & 1 & 1 & 1 & 1 & 1 & 1 & 1 & 1 & 1 \\
\hline
\end{tabular}

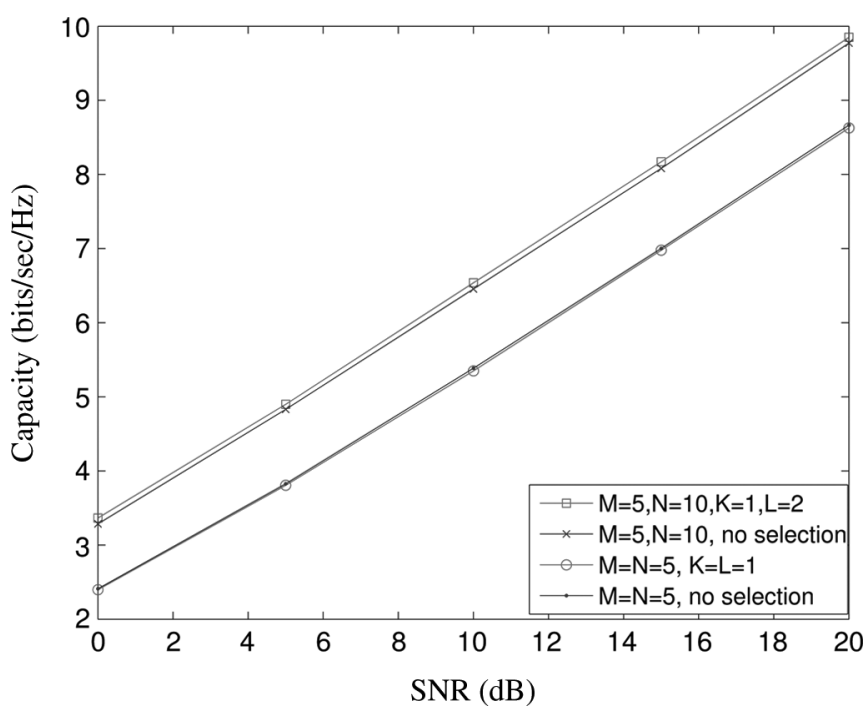

Fig. 1. Ergodic capacity of the keyhole channel with and without antenna selection.

As mentioned earlier, we wish to find the minimum number of selected receive antennas such that the equivalent SNR is no less than $E[\gamma]$. Considering that $g(L)$ above is a monotonic function, the number of selected antennas will be

$$
\hat{L}=\min g^{-1}(\tilde{\gamma}) \quad \text { subject to } \tilde{\gamma} \geq \gamma .
$$

Table I shows the calculated values of $\hat{L}$ for various systems. It is interesting to observe that across a large group of systems, a small number of selected antennas is sufficient to match the capacity of a baseline system with no transmit CSI, but with full hardware at both sides. Antenna selection requires a small amount of feedback, but has dramatically smaller hardware requirements.

Fig. 1 shows the ergodic capacity of the keyhole channel for two cases: $M=N=5$, out of which a $1 \times 1$ system is chosen; and $M=5, N=10$, out of which a $1 \times 2$ system is chosen. Capacity is compared between the antenna selection and the full system. The Monte Carlo simulation is performed over 5000 independent channel realizations. The simulation results show that at the cost of a few bits of feedback, a keyhole channel can be reduced to a low-order SIMO channel without a considerable loss in the capacity. 

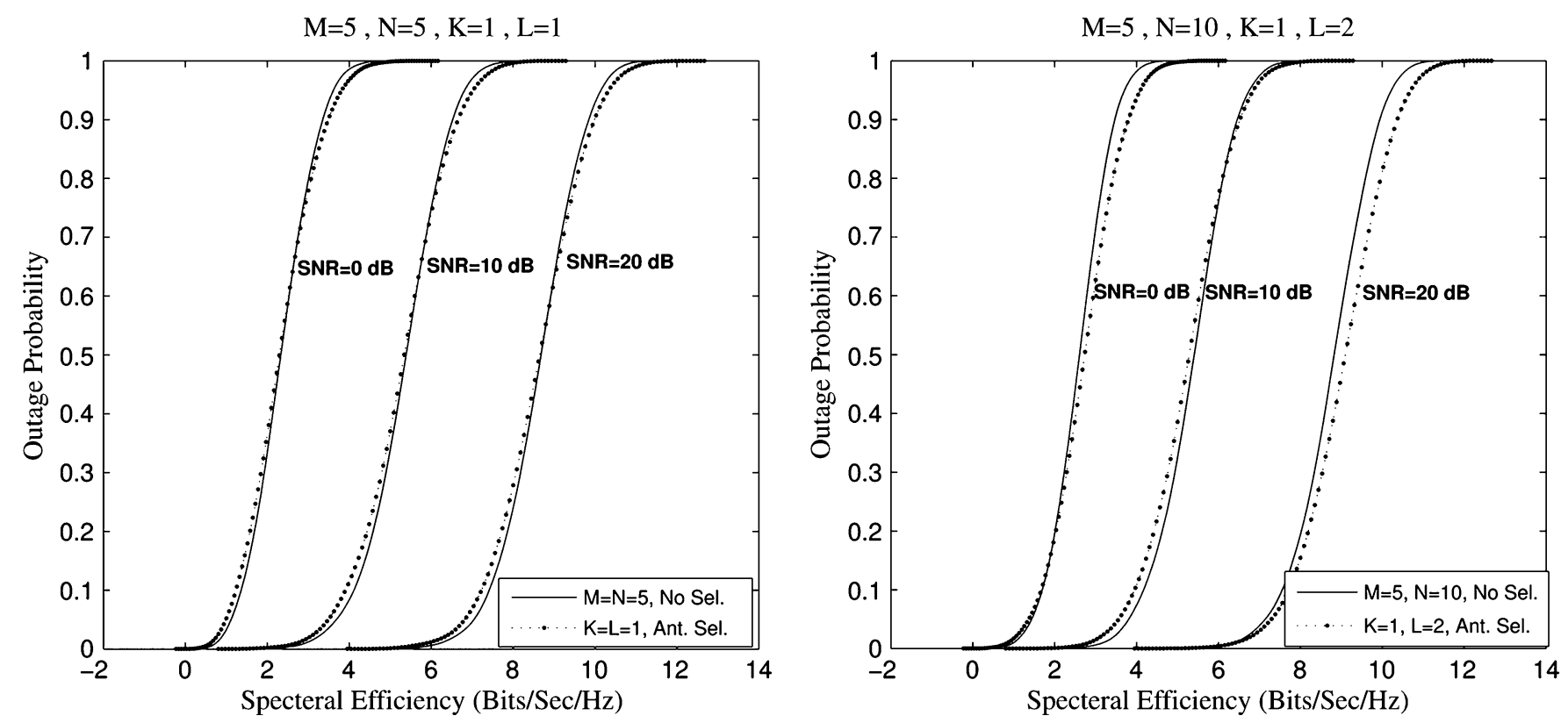

Fig. 2. Outage capacity of the keyhole channel with and without antenna selection.

Fig. 2 shows the outage probability of the keyhole channel in two cases: $M=N=5$, out of which a $1 \times 1$ system is chosen; and $M=5, N=10$, out of which a $1 \times 2$ system is chosen. Simulations show that in the keyhole channel, the outage probability of the selection channel is very close to the outage probability of the full system.

\section{DIVERSITY ORDER}

It has already been stated [6] and proved [7] that the diversity order of the keyhole channel is $\min (M, N)$. In this section, we prove that antenna selection has no impact on the diversity order of the keyhole channel. The outage probability for the keyhole channel is

$$
\begin{aligned}
P_{\text {out }} & =\operatorname{Pr}[\mathcal{I}<R]=\operatorname{Pr}[\log (1+\rho \gamma)<R] \\
& =\operatorname{Pr}\left[\gamma<\frac{2^{R}-1}{\rho}\right]=F_{\gamma}\left(\frac{2^{R}-1}{\rho}\right)
\end{aligned}
$$

where $F_{\gamma}(\cdot)$ is the cumulative distribution function $(\mathrm{CDF})$ of the random variable $\gamma$. The diversity order is defined as

$$
d=\lim _{\rho \rightarrow \infty} \frac{-\log P_{\text {out }}}{\log \rho}
$$

therefore, (11) suggests that the diversity order is equal to the exponent of the lowest order term in the asymptotic expansion of the $F_{\gamma}(a)$ for small enough $a$, where $a \triangleq\left(2^{R}-1\right) / \rho$, i.e., $F_{\widetilde{\gamma}}(a)=a^{d}+o\left(a^{d}\right)$.

Theorem 1: The diversity order of the keyhole channel with antenna selection is $\min (M, N)$. In particular, the outage probability with antenna selection has the following asymptotic behavior:

$$
P_{\text {out }}(a) \stackrel{\circ}{=} \begin{cases}a^{N}, & N<M \\ a^{M}, & N>M \\ a^{N} \log \left(\frac{1}{a}\right), & N=M .\end{cases}
$$

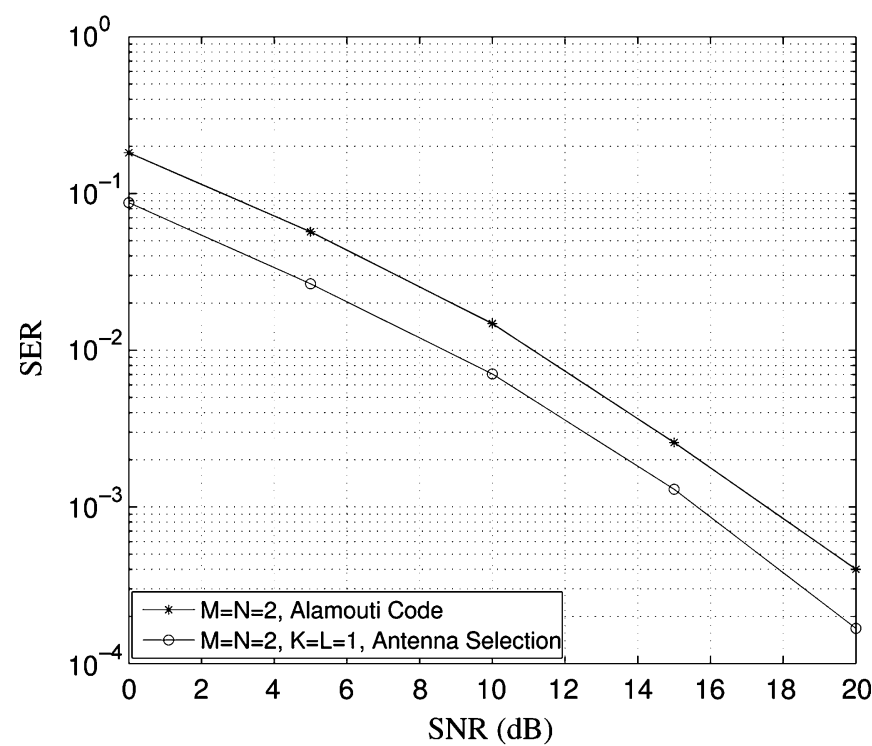

Fig. 3. Symbol-error rate versus SNR for binary phase-shift keying.

Proof: See the Appendix.

Notice that when $M=N$, then the diversity behavior can not be described by an integer. For such a case, the keyhole channel behaves better than a channel of diversity order $N-1$, but its performance is not as good as a channel with diversity $N$. This is exactly the same result obtained in [7].

To demonstrate the diversity order of the antenna-selection channel, we refer the reader to Fig. 3, which depicts the symbolerror rate of an uncoded keyhole channel with two transmit and two receive antennas $(M=N=2)$. We show the performance of Alamouti code using $2 \times 2$ antennas, versus an antenna-selection system that at each point in time selects down to a SISO system $(1 \times 1)$. Both have diversity two, but we also see the the logarithmic penalty predicted by [7] and Theorem 1 . Antenna 
selection provides $2 \mathrm{~dB}$ gain over the Alamouti space-time code (which is open loop). Antenna selection requires a feedback of $\log M=1$ bit per fading state, to select the best transmit antenna. Since the fading states vary much slower than the symbol transmission rate, the equivalent feedback rate is small.

\section{CONCLUSION}

In this letter, we study antenna-selection channels in the presence of the keyhole condition, and develop two results. First, we analyze the capacity of antenna selection under the keyhole condition and show that only a very small number of antennas need be selected (only one at transmitter, and very few at receiver) to match the performance of a baseline system that operates with all antennas. Second, we prove that antenna selection does not change the diversity order of the keyhole channel.

\section{APPENDIX}

Lemma 1: In the asymptote of small $a$ we have

$$
\int_{0}^{\infty} \frac{e^{-\left(x+\frac{a}{x}\right)}}{x^{\nu+1}} d x \stackrel{\circ}{=} \begin{cases}\frac{\Gamma(\nu)}{a^{\nu}}, & \nu>0 \\ -\log a, & \nu=0 \\ \Gamma(-\nu), & \nu<0 .\end{cases}
$$

Proof: We have

$$
\int_{0}^{\infty} \frac{e^{-\left(x+\frac{a}{x}\right)}}{x^{\nu+1}} d x=\frac{2}{a^{\frac{\nu}{2}}} K_{\nu}(2 \sqrt{a})
$$

where $K_{\nu}(\cdot)$ is the modified Bessel function of the second kind [8]. For small $z$, the following asymptotic formulae hold [8, p. 375 , eq. 9.6.6, 9.6.8, 9.6.9]:

$$
K_{\nu}(z) \stackrel{\circ}{=} \begin{cases}\frac{1}{2} \Gamma(\nu)\left(\frac{z}{2}\right)^{-\nu}, & \nu>0 \\ -\log \frac{z}{2}, & \nu=0 \\ \frac{1}{2} \Gamma(-\nu)\left(\frac{z}{2}\right)^{\nu}, & \nu<0 .\end{cases}
$$

Now let $z=2 \sqrt{a}$, combining (14) and (15), we arrive at (13).

Proof (Theorem 1): We have $\max _{i}\left\{\left|\mathbf{h}_{r}(i)\right|^{2}\right\} \leq\left\|\widetilde{\mathbf{h}}_{r}\right\|^{2} \leq$ $\left\|\mathbf{h}_{r}\right\|^{2}$, hence

$$
\log (1+\rho \hat{\gamma}) \leq \log (1+\rho \widetilde{\gamma}) \leq \log (1+\rho \gamma)
$$

where

$$
\begin{aligned}
\hat{\gamma} & =\left(\max _{i}\left|\mathbf{h}_{t}(i)\right|^{2}\right)\left(\max _{j}\left|\mathbf{h}_{r}(j)\right|^{2}\right) \\
\widetilde{\gamma} & =\left\|\widetilde{\mathbf{h}}_{r}\right\|^{2}\left(\max _{i}\left|\mathbf{h}_{t}(i)\right|^{2}\right) \\
\gamma & =\left\|\mathbf{h}_{t}\right\|^{2}\left\|\mathbf{h}_{r}\right\|^{2} .
\end{aligned}
$$

Thus for all $a \geq 0$

$$
F_{\widehat{\gamma}}(a) \leq F_{\gamma}(a) \leq F_{\gamma}(a) .
$$

It was shown in [7] that the diversity order of the keyhole channel (corresponding to $\gamma$ ) is $\min (M, N)$. We only need to prove a similar result for the lower bound $\widetilde{\gamma}$, which corresponds to antenna selection with $K=L=1$. In other words, it suffices to prove that for the keyhole channel, selecting the best antennas at both the transmit and receive sides provides full diversity. $\widehat{\gamma}$ is the product of two independent random variables $\widehat{\gamma}_{1}=\max _{i}\left|\mathbf{h}_{t}(i)\right|^{2}$ and $\widehat{\gamma}_{2}=\max _{i}\left|\mathbf{h}_{r}(i)\right|^{2}$, each the extreme value of exponentially distributed independent random variables. Hence, the probability density function (pdf) of $\widehat{\gamma}_{1}$ and $\widehat{\gamma}_{2}$ (denoted by $f_{1}(\cdot)$ and $f_{2}(\cdot)$, respectively) are given by

$$
\begin{aligned}
& f_{1}(x)=M e^{-x}\left(1-e^{-x}\right)^{M-1} \\
& f_{2}(x)=N e^{-x}\left(1-e^{-x}\right)^{N-1} .
\end{aligned}
$$

The pdf of $\widehat{\gamma}$ can be calculated as follows:

$$
\begin{aligned}
f_{\widehat{\gamma}}(a) & =\int_{0}^{\infty} f_{1}(x) f_{2}\left(\frac{a}{x}\right) \frac{d x}{x} \\
& =\int_{0}^{\infty} f_{2}(x) f_{1}\left(\frac{a}{x}\right) \frac{d x}{x} .
\end{aligned}
$$

Case I $(M>N)$ : We use (19)

$f_{\widehat{\gamma}}(a)=M N \int_{0}^{\infty} e^{-\left(x+\frac{a}{x}\right)}\left(1-e^{-x}\right)^{M-1}\left(1-e^{-\frac{a}{x}}\right)^{N-1} \frac{d x}{x}$.

Applying the Taylor series expansion

$$
\begin{aligned}
(1 & \left.-e^{-x}\right)^{M-1}\left(1-e^{-\frac{a}{x}}\right)^{N-1} \\
& =\left(x-\frac{x^{2}}{2 !}+\cdots\right)^{M-1}\left(\frac{a}{x}-\frac{a^{2}}{2 ! x^{2}}+\cdots\right)^{N-1} \\
& =a^{N-1} x^{M-N}\left(1+\sum_{j \geq 0, k \leq j} c_{j k} a^{j} x^{k}\right) .
\end{aligned}
$$

By substitution, (21) can be calculated via term-by-term integration. This is due to the dominated convergence theorem, since the terms in (22) are bounded above by one. It follows that

$$
f_{\widehat{\gamma}}(a)=I_{1}(a)+I_{2}(a)
$$

where

$$
I_{1}(a)=M N a^{N-1} \int_{0}^{\infty} \frac{e^{-\left(x+\frac{a}{x}\right)}}{x^{N-M+1}} d x
$$

and

$$
I_{2}(a)=M N \sum_{j \geq 0, k \leq j} c_{j k} a^{N+j-1} \int_{0}^{\infty} \frac{e^{-\left(x+\frac{a}{x}\right)}}{x^{N-M-k+1}} d x .
$$

From Lemma 1, $I_{1}(a) \stackrel{\circ}{=} a^{N-1}$; also each term in the sum in (25) is $O\left(a^{N+p-1}\right)$ for some $p \in\{0,1,2, \ldots\}$. Since $I_{2}(a)$ converges, we conclude that $I_{2}(a) \stackrel{\circ}{=} a^{N-1}$, thus $f_{\widehat{\gamma}}(a) \stackrel{\circ}{=} a^{N-1}$. On the other, using L'Hopital's rule, we know that in the asymptote of small $a$, we have

$$
F_{\widehat{\gamma}}(a) \stackrel{\circ}{=} a f_{\widehat{\gamma}}(a)
$$


Thus, when $M>N$, we have $F_{\widehat{\gamma}}(a) \stackrel{\circ}{=} a^{N}$, i.e., the diversity order is $N=\min (M, N)$.

Case II $(N>M)$ : In this case, we use (20). Considering the symmetry of the equation with respect to $M$ and $N$, the problem is reduced to Case I with $M$ and $N$ interchanged, thus, the diversity order again is $M=\min (M, N)$.

Case III $(N=M)$ : Using Lemma 1 and an argument similar to Case I, we have $f_{\widehat{\gamma}}(a) \stackrel{\circ}{=} a^{N-1} \log (1 / a)$. Thus $F_{\widehat{\gamma}}(a) \stackrel{\circ}{=}$ $a^{N-1} \log (1 / a)$

$$
F_{\widehat{\gamma}}(a) \stackrel{\circ}{=} \begin{cases}a^{N}, & N<M \\ a^{M}, & N>M \\ a^{N} \log \left(\frac{1}{a}\right), & N=M .\end{cases}
$$

But this is exactly the same as the asymptotic behavior of $F_{\gamma}(\cdot)$ for a general keyhole channel [7]. In other words, $F_{\widehat{\gamma}}(a) \stackrel{\circ}{=} F_{\gamma}(a)$. Thus (17) necessitates that $F_{\widetilde{\gamma}}(a)$ also has the same asymptotic behavior, and this proves the theorem.

\section{REFERENCES}

[1] D. Chizhik, G. J. Foschini, M. J. Gans, and R. A. Valenzuela, "Keyholes, correlations, and capacities of multielement transmit and receive antennas," IEEE Trans. Wireless Commun., vol. 1, no. 2, pp. 361-368, Apr. 2002.

[2] H. Shin and J. H. Lee, "Capacity of multiple-antenna fading channels: Spatial fading correlation, double scattering, and keyhole," IEEE Trans. Inf. Theory, vol. 49, no. 10, pp. 2636-2647, Oct. 2003.

[3] P. Almers, F. Tufvesson, and A. Molisch, "Measurement of keyhole effect in a wireless multiple-input multiple-output (MIMO) channel," IEEE Commun. Lett., vol. 7, no. 8, pp. 373-375, Aug. 2003.

[4] S. Sanayei and A. Nosratinia, "Antenna selection in MIMO systems," IEEE Commun. Mag., 42, no. 10, pp. 68-73, Oct. 2004.

[5] M. Z. Win and J. H. Winter, "Analysis of hybrid selection/maximalratio combining in Rayleigh fading," IEEE Trans. Commun., vol. 47, no. 12, pp. 1773-1776, Dec. 1999.

[6] D. Gesbert, H. Bolcskei, D. A. Gore, and A. J. Paulraj, "Outdoor MIMO wireless channels: Models and performance prediction," IEEE Trans. Commun., vol. 51, no. 12, pp. 1926-1934, Dec. 2003.

[7] S. Sanayei and A. Nosratinia, "Space-time codes in keyhole channels: Analysis and design issues," in Proc. IEEE Global Telecommun. Conf., Dallas, TX, Dec. 2004, vol. 6, pp. 3768-3772.

[8] M. Abramowitz and A. I. Stegun, Handbook of Mathematical Functions. New York: Dover, 1972. 